\title{
A novel surgical method for treating medial-end clavicle fractures
}

\author{
WEN-PENG XIE ${ }^{1 *}$, YONG-KUI ZHANG ${ }^{2 *}$, YAN-HUA CHEN ${ }^{2}$, SHI-LU WANG ${ }^{1,2}$, \\ HONG-HAO XU ${ }^{2}$ and RONG-XIU BI ${ }^{1,2}$ \\ ${ }^{1}$ First Clinical Medical College, Shandong University of Traditional Chinese Medicine, Jinan, Shandong 250355; \\ ${ }^{2}$ Department of Orthopedics, Affiliated Hospital of Shandong University of Traditional Chinese Medicine, \\ Jinan, Shandong 250014, P.R. China
}

Received March 8, 2018; Accepted August 9, 2018

DOI: $10.3892 /$ etm.2018.6870

\begin{abstract}
The present study examined the effectiveness of the method of inversing the distal clavicle anatomic locking plate to treat medial-end clavicle fractures. This involved retrospectively analyzing six cases of medial-end clavicle fractures treated using the method of inversing the distal clavicle anatomic locking plate between September 2013 and December 2015. These cases included five men and one woman with an average age of $46.3 \pm 10.6$ years old and a range of 24-66 years old. Among these cases, one was type A, two were type $\mathrm{C}$, and three were type $\mathrm{D}$, based on the Throckmorton type classification. All patients had fresh closed fractures, which were caused by direct injury. A regular postoperative follow-up was performed and the therapeutic efficacy was evaluated systematically with reference to the Rockwood and Disability of the Arm, Shoulder and Hand (DASH) score standards. All six cases had a follow-up visit, which was between 10 and 14 months with an average of $12.0 \pm 2.2$ months. All patients exhibited osseous healing and functional recovery without incision infection, nerve vascular injury, chest injury, fracture nonunion, or other complications. According to the Rockwood and DASH score standards, evaluation of the curative effect showed that five cases were excellent and one case was good, and the associated rate for achieving good or excellent outcomes was $100 \%$. All of the DASH scores were $<10$. These results indicated that the effect of the treatment of medial-end clavicle fractures by the method of inversing the distal clavicle anatomic locking plate was reliable, and the functional recovery of patients was good, making this method worthy of clinical promotion.
\end{abstract}

Correspondence to: Dr Rong-Xiu Bi, Department of Orthopedics, Affiliated Hospital of Shandong University of Traditional Chinese Medicine, 16369 Jingshi Road, Jinan, Shandong 250014, P.R. China E-mail: birongxiu@163.com

${ }^{*}$ Contributed equally

Key words: medial-end clavicle fractures, surgical method, inverse distal clavicle anatomic locking plate, Rockwood and disability of the Arm, shoulder and hand score standard, effectiveness

\section{Introduction}

Clavicle fractures are common in clinics and the incidence rate is $~ 2.6-12 \%$ of all fractures (1). Allman (2) divided clavicle fractures into three types: The distal clavicle fracture, the middle clavicle fracture, and the medial-end clavicle fracture. Among these, medial-end clavicle fractures are rare and make up only $2-10 \%(3-6)$ of all clavicle fractures; this type of fracture is usually caused by direct, high-energy injury. The medial-end clavicle fracture is rare and conservative treatment is generally used in view of the complex anatomy of the proximal clavicle and the risk of injury to important neurovascular organs, however, the effect of conservative treatment is not ideal, which has led to increasing focus on the potential of surgical treatment. However, there are no unified recognized standards or specifications for the methods and materials of internal fixation. In addition, there is no fixation material specifically designed for medial-end clavicle fractures. Currently, internal fixators for proximal clavicle fractures include Kirschner wire, steel wire, screws and various types of steel plates.

The shape of the inverse distal clavicle anatomic locking plate is similar to the form of the medial-end clavicle. The design of double row locking holes in the distal part of the plate can fix more layers of cortex and strengthen the stability of fixation. Additionally, the plate can satisfactorily adhere to the bone through molding. Inspired by this, the present study treated medial-end clavicle fractures using a method of inversing the distal clavicle anatomic locking plate. The therapeutic effects were found to be satisfactory.

\section{Patients and methods}

A total of six cases of medial-end clavicle fracture were treated by the method of inverting the distal clavicle anatomic locking plate between September 2013 and December 2015 at the Affiliated Hospital of Shandong University of Traditional Chinese Medicine (Jinan, China). All patients signed written informed consent prior to enrolment in the study and the cases conformed to the surgical indication of a displacement of $>1 \mathrm{~cm}$ between the fragment ends. The patient demographics are summarized in Table I. The patients were classified based on the Throckmorton type (5). All cases were freshly-closed fractures and were caused by direct injury. Regular postoperative follow-ups were performed and the therapeutic efficacy 
was systematically evaluated by referencing the Rockwood score standard and the Disability of the Arm, Shoulder and Han (DASH) score standard $(7,8)$.

When the patients were hospitalized, preoperative routine examinations were performed. First of all, the relevant departments were consulted to actively treat medical diseases and eliminate the contraindication of surgery due to presentation of coronary heart disease, hypertension or diabetes. Subsequently, the surgical plan was developed following department discussion, and antibiotics were administered to patients $30 \mathrm{~min}$ prior to surgery. Finally, surgery was performed for each patient when all these preparations had been completed.

A circuit nurse performed urethral catheterization for the patient, and the patient was then placed in the beach chair position to adequately expose the surgical area following the successful administration of brachial plexus anesthesia (with local anesthesia when necessary). The skin, subcutaneous tissue, and fascia were cut in turn, and the periosteum was finitely stripped subsequent to making an 8-10 cm skin incision. The incision accorded to the direction of clavicle centered on the fracture end. The fracture was reset anatomically, following which the inversely implanted distal clavicle anatomic locking plate (Jiangsu Hopromed Co., Ltd., Jiangsu, China; http://www. js-hp.com) was used to fix the broken fracture end. The plate was carefully shaped based on the different clavicle forms so it adhered to the surface of the bone without a gap. The broken fracture end of type D was first fixed using a lag screw (Jiangsu Hopromed Co., Ltd.). Three locking screws (Jiangsu Hopromed Co., Ltd.) were placed on the far-end of the fracture and 2-3 locking screws were placed on the near-end (Fig. 1A-C). The plate was implanted across the sternoclavicular joint when there was insufficient space in the near-end of the fracture to implant 2-3 locking screws. Two locking screws were placed on the manubrium sterni in order to enhance firmness and stability in this situation. Care was required to drill holes and avoid the screws passing through the contralateral cortex, as the heart, vessels and other important organs are located in the deep of manubrium sterni. Unstable fracture fragments were bundled with double surgical sutures, and artificial bone (Shanghai Anjiu Biotechnology Co., Ltd., Shanghai, China) was grafted in the bone defection to accelerate fracture healing. An X-ray examination was performed to appraise the reduction and the length of the screws. Finally, the incision was closed in the usual manner.

Postoperatively, all patients were given antibiotics (Cefotiam, Zhejiang Yongning Pharmaceutical Co., Ltd., Taizhou, China) only once to prevent infection, and the extremity of the forearm was immobilized in a sling for 2 weeks. This surgical method allowed excellent fixation and early mobilization under the guidance of the doctor. Patients gradually performed exercises using the elbow, wrist and hand as comfort would allow. After 4 weeks, the patients began to activate the shoulder in all directions and progressively increased the range. Normal activities, including sports and weight-bearing exercises, were not allowed until fracture union had been completed. Postoperative follow-up was periodically performed to evaluate the shoulder function and observe complications including incision infection, nerve vascular injury, chest injury and nonunion. A final assessment of shoulder function was assessed based on the Rockwood
Table I. Select patient demographics and operative outcomes.

\begin{tabular}{lc}
\hline Characteristic & $\mathrm{n}$ \\
\hline Total number of patients & 6 \\
Average age, years (range) & $46.3 \pm 10.6$ (24-66) \\
Sex & 5 \\
Male & 1 \\
Female & \\
Affected side & \\
Left & 2 \\
Right & 4
\end{tabular}

Throckmorton type

Type A

1

Type B

0

Type C

2

Type D

3

Type E

Average length of follow-up, months (range)

Mean fluoroscopic frequency, (range)

Mean operative time, min (range)

Average time to healed X-ray, months (range)

$12.0 \pm 2.2(12-14)$

$48.4 \pm 6.7(45-65)$

$3.5(2.9-6.2)$

Functional recovery

$\begin{array}{ll}\text { Excellent } & 5 \\ \text { Good } & 1 \\ \text { General } & 0 \\ \text { Poor } & 0\end{array}$

DASH score (range) $\quad 8.6 \pm 1.2(7-9)$

DASH, Disability of the Arm, Shoulder and Hand.

score standard and DASH score standard. The Rockwood score ranges between 0 and 15 with values 13-15 representing excellent, 10-12 representing good, 7-9 representing general, and $<7$ representing poor. The DASH score ranges between 0 and 100 with a higher score indicating a higher level of functional disability.

The institutional review boards and ethics committees of the Affiliated Hospital of Shandong University of Traditional Chinese Medicine approved the present study. All six patients provided consent for the publication of the present report.

\section{Results}

In the six cases, none of the patients had their sternoclavicular joint fixed; it was preferable to avoid fixing the sternoclavicular joint, if possible, as it is a fretting joint. The six patients received a postoperative follow-up, which ranged between 10 and 14 months with an average of 12 months. Osseous healing and functional recovery were observed (Fig. 1D) without incision infection, nerve vascular injury, chest injury, nonunion or other complications. According to the Rockwood and DASH score standards, evaluation of the curative effect showed that five cases were 'excellent' and one case was 

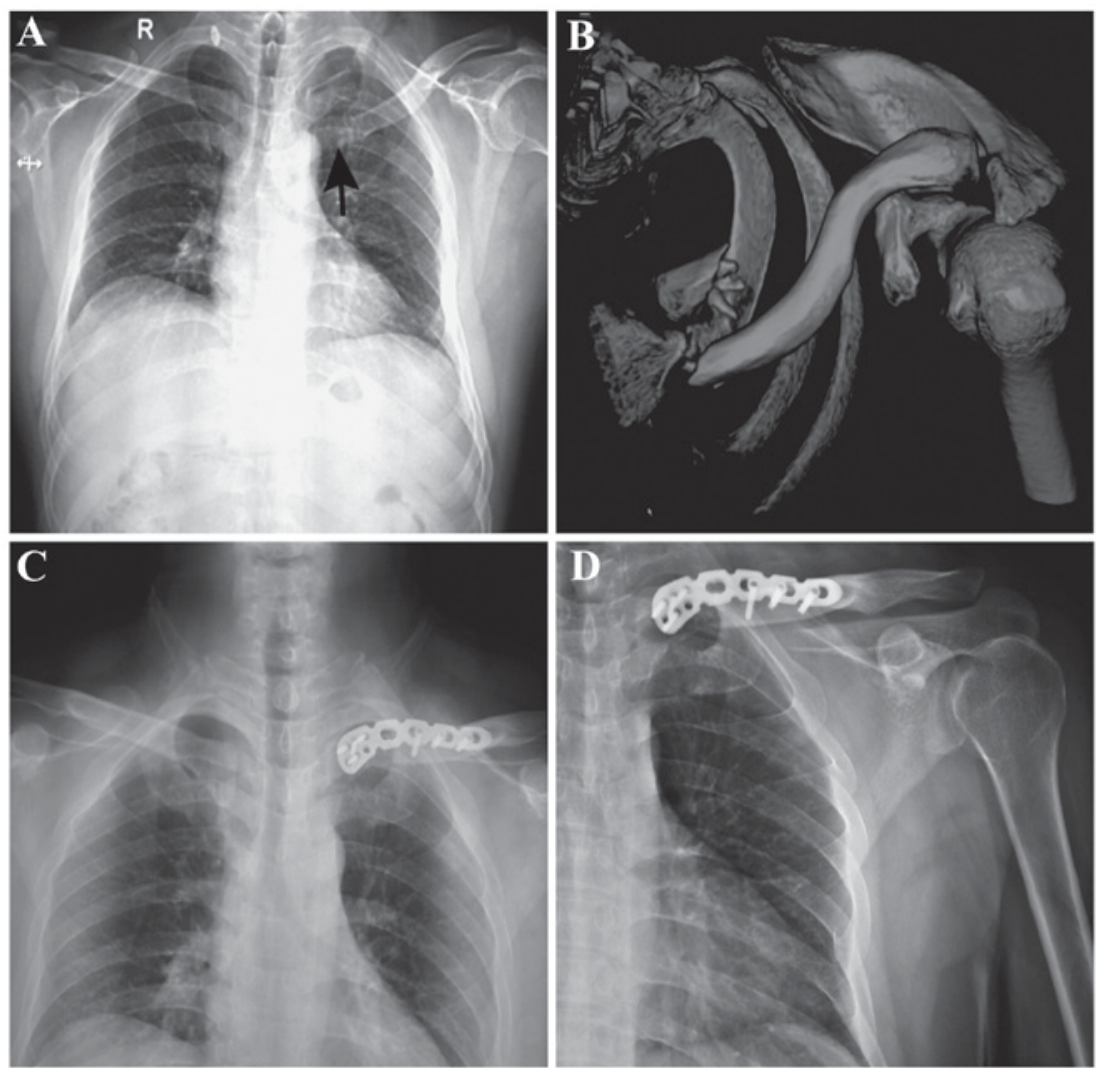

Figure 1. Radiographic findings. (A) Preoperative radiograph (left displaced medial end clavicle fracture). (B) CT scan with three-dimensional reconstruction demonstrated comminuted extra-articular fracture with posterior displacement of the medial end. (C) Postoperative radiograph. The fracture was fixed with an inverse distal clavicle anatomic locking plate. (D) Radiograph at 1 year post-surgery. The fracture showed osseous healing without malformation.

'good', with an associated rate for achieving good or excellent outcomes of $100 \%$. All of the DASH scores were $<10$ and the average score was $8.6 \pm 1.2$.

\section{Discussion}

The medial-end clavicle fracture is a relatively rare injury and accounts for only $2-10 \%$ of all clavicle fractures. Throckmorton and Kuhn (5) divided medial-end clavicle fractures into five types: Type A (transverse fracture), type B (oblique intra-articular fracture), type C (oblique extra-articular fracture), type D (comminuted fracture), and type $\mathrm{E}$ (avulsion fracture). These fractures have traditionally been treated conservatively to avoid damage to the deep tissues during surgery, including the brachial plexus, subclavian vein, axillary vein, and apex pulmonis. However, conservative treatment of these fractures can lead to serious complications, including nonunion, malunion, and functional disability (9). With continuous improvement at the clinical level, surgical methods, including open reduction and internal fixation, partial claviculectomy, and fusion of the sternoclavicular joints, have been reported in the available literature $(3,5,10,11)$. Due to the high rate of the postoperative complications, partial claviculectomy and fusion of the sternoclavicular joints have gradually been avoided. By contrast, open reduction and internal fixation have been considered to be the preferred method (12). However, there are no unified recognized standards or specifications for the methods and materials of the internal fixation. In addition, there is no fixation material specifically designed for medial-end clavicle fractures.
Bartonícek et al (13) reported that they obtained a good curative effect by using cerclage wires to treat five cases, and used the visual analogue scale score and the DASH score to evaluate the effects. In another study, Oe et al concluded that the locking T-plate and DCP plate were effective (14). They treated 10 medial-end clavicle fractures and all patients gained osseous healing without complications. Correa et al (15) showed that using a reconstruction plate to treat medial-end clavicle fractures made it possible to obtain satisfactory clinical effects. Sidhu et al, Ding et al and Sloan et al used clavicular hook plates and contralateral lateral clavicle locking plates to treat medial-end clavicle fractures $(4,16,17)$. All achieved good effects on postoperative follow-up. These materials for internal fixation were effective, however, the sample size was small and no control study was designed in these clinical reports. In addition, the fixed strength and stability of the T-plate are relatively poor and it is easy to cause stress concentration around the sternoclavicular joint, causing plate fracture. The clavicular hook plate has poor ability to resist distortion and it is easy to penetrate through the sternum; the hook penetrating through the sternum will damage the internal organs. The fixation strength of Kirschner wire and cerclage wire are too weak to fix fractures. In addition, full exposure in the surgical field is required when using reconstruction plate to treat fractures, and the plate is too difficult to shape. Accordingly, the reconstruction plate may damage the blood supply and extend surgery time.

In the present study, six cases of medial-end clavicle fractures were treated using a method of inverting the distal 
clavicle anatomic locking plate, and satisfactory clinical effects were obtained. It is important to pay attention to the following points during surgery: Firstly, adequate locking screws require placement on the far-end and near-end of the fracture to ensure stability; the plate is implanted across the sternoclavicular joint when there is insufficient space in the near-end of the fracture to implant 2-3 locking screws; 1-2 locking screws are placed on the manubrium sterni in order to enhance firmness and stability in this particular situation; care is required in drilling holes and to avoid the screws passing through the contralateral cortex as the heart, vessels and other important organs are located in the deep of manubrium sterni. Secondly, the broken fracture end of type $\mathrm{C}$ and type $\mathrm{D}$ fractures require fixation using a lag screw, with unstable fracture fragments bundled with double surgical sutures. These methods can increase the strength of the fixation and promote fracture healing. Thirdly, it is recommended that artificial bone is grafted in the bone defection to accelerate fracture healing. Finally, it is necessary to remove this plate as soon as possible following fracture healing in order to avoid fracture and reject reaction of plate.

In conclusion, the clinical effect of the treatment of medial-end clavicle fractures using the method of inverting the distal clavicle anatomic locking plate was reliable and the functional recovery of patients was good. However the sample size was small and no control study was designed in the present report to support the conclusion, therefore, the results only provide a reference for the treatment. It is necessary to design a specific locking plate for medial-end clavicle fractures. Therefore, this method is worthy of further promotion, and the development of a specific locking plate for medial-end clavicle fracture is required.

\section{Acknowledgements}

The authors thank Ms. Xiaodi Li, Nanjing University of Traditional Chinese Medicine (Nanjing, China) for her linguistic advice.

\section{Funding}

Not applicable.

\section{Availability of data and materials}

The datasets used and/or analysed during the current study are available from the corresponding author on reasonable request.

\section{Authors' contributions}

W-PX and Y-KZ performed the postoperative follow-up and were major contributors in writing the manuscript. R-XB guided the design and implementation of the surgery. Y-HC analysed and interpreted the data. S-LW and $\mathrm{H}-\mathrm{HX}$ took part in the surgery and processed the images in the manuscript. All authors read and approved the final manuscript.
Chinese Medicine (Nanjing, China) approved the present study.

\section{Patient consent for publication}

All six patients provided consent for the publication of the present report.

\section{Competing interests}

The authors declare that they have no competing interests.

\section{References}

1. Kihlström C, Möller M, Lönn K and Wolf O: Clavicle fractures: Epidemiology, classification and treatment of 2422 fractures in the Swedish Fracture Register; an observational study. BMC Musculoskelet Disord 18: 82, 2017.

2. Allman FL Jr: Fractures and ligamentous injuries of the clavicle and its articulation. J Bone Joint Surg Am 49: 774-784, 1967.

3. Smelt J, Khakha RS, Harrison-Phipps K, Richards A and Bille A: An isolated traumatic medial third clavicular fracture requiring surgical fixation. Ann Thorac Surg 103: e297-e298, 2017.

4. Sidhu VS, Hermans D and Duckworth DG: The operative outcomes of displaced medial-end clavicle fractures. J Shoulder Elbow Surg 24: 1728-1734, 2015.

5. Throckmorton T and Kuhn JE: Fractures of the medial end of the clavicle. J Shoulder Elbow Surg 16: 49-54, 2007.

6. Vander Meijden OA, Gaskill TR and Millett PJ: Treatment of clavicle fractures: Current concepts review. J Shoulder Elbow Surg 21: 423-429, 2012.

7. Shetty SK, Chandran R, Ballal A, Mathias LJ, Hegde A and Shetty A: To operate or not to operate the mid-shaft fractures of the clavicle: A comparative study of functional outcomes of the two methods of management. J Clin Diagn Res 11: RC01-RC03, 2017.

8. Rockwood CA Jr, Groh GI, Wirth MA and Grassi FA: Resection arthroplasty of the sternoclavicular joint. J Bone Joint Surg Am 79: 387-393, 1997.

9. Poggetti A, Novi M, Rosati M, Battistini P, Parchi P and Lisanti M: Unusual medial-end clavicle fracture combined with double disruption of the superior shoulder suspensory complex (SSSC): A case report in triathlon Athlete. J Orthop Case Rep 6: 19-21, 2016.

10. Bourghli A and Fabre A: Proximal end clavicle fracture from a parachute jumping injury. Orthop Traumatol Surg Res 98: 238-241, 2012.

11. Teng HG and Liu AL: Partial claviculectomy after non-union of proximal clavicle fracture. BMJ Case Rep 2013: pii: bcr2013008874, 2013.

12. Koch MJ and Wells L: Proximal clavicle physeal fracture with posterior displacement: Diagnosis, treatment, and prevention. Orthopedics 35: e108-e111, 2012.

13. Bartonícek J, Fric V and Pacovsk V: Displaced fractures of the medial end of the clavicle: Report of five cases. J Orthop Trauma 24: e31-e35, 2010

14. Oe K, Gaul L, Hierholzer C, Woltmann A, Miwa M, Kurosaka M and Buehren V: Operative management of periarticular medial clavicle fractures: Report of 10 cases. J Trauma 72: E1-E7, 2012.

15. Correa MC, Gonçalves LB, Vilela JC, Leonel IL, Costa LP and de Andrade RP: Extra-articular fracture of the medial end of the clavicle associated with type IV acromioclavicular dislocation: Case report. Rev Bras Orthop 46: 596-601, 2011.

16. Ding M, Ni J, Hu J and Song D: Rare complication of clavicular hook plate: Clavicle fracture at the medial end of the plate. J Shoulder Elbow Surg 20: e18-e20, 2011.

17. Sloan AG, Howcroft D and Wykes PR: Operative treatment of medial clavicle fractures: an alternative suigicle technique. Injury Extra 39: 270-272, 2008.

\section{Ethics approval and consent to participate}

The institutional review boards and ethics committees of the Affiliated Hospital of Shandong University of Traditional 